\title{
含整体单极子的黑洞外部时空中的陀螺 自转轴的进动效应*
}

\author{
荆继良 余洪伟 王永久 \\ (长沙铁道学院理论天体物理研究室, 长沙 410075)
}

\section{关链词黑洞、陀螺、整体单极子}

早期宇宙中真空相变可导致诸如单极子、宇宙弦一类的拓扑缺陷 ${ }^{[11}$, 这些缺陷的出现与周 域或整体对称破缺有关. 单极子和宇宙弦的出现可能会在宇宙中引起一些重要的、可观测的 效应, 也许对星系的形成产生影响 ${ }^{[1,2]}$. 近来人们对单极子的研究十分感兴趣. Barriola 和 Vilenkin ${ }^{[3]}$ 得到了在 $O(3)$ 中整体单极子引力场. 我们研究了现实局域单极子引力 场 ${ }^{[4-\eta]}$. 文献 [8]讨论了在宇宙相变过程中由于整体单极子的形成而导致的量子引力粒子产生效应. 文 献 [9]研究了整体单极子时空中的真空极化效应. 文献 [10]和 [3]指出, 当一个黑洞吞食了整 体单极子,其外部时空度规可写为

$$
\begin{aligned}
d S^{2}- & \left(1-8 \pi G \eta^{2}-\frac{2 G M}{r}\right) d t^{2}-\frac{d r^{2}}{1-8 \pi G \eta^{2}-\frac{2 G M}{r}} \\
& -r^{2}\left(d \theta^{2}+\sin ^{2} \theta \mathrm{d} \varphi^{2}\right),
\end{aligned}
$$

式中 $M$ 为黑洞的质量, $\eta$ 为对称破缺标度. 如果吞食整体单极子的黑洞质量远大于 $1 / G \eta$, 那 么, Schwarzschild 半径就远大于整体单极子核的半径. 他们在文献 [10]中还讨论了由 (1) 式给出的时空中光线偏转等一些引力性质.

早在 60 年代, 为了检验 Einstein 的广义相对论理论, Schiff ${ }^{[1]}$ 研究了引力场中陀螺运 动, Everitt ${ }^{[2]}$ 等人进行了实验验证. 本文采用 Schiff、Gonzalez-Martin ${ }^{[13,14]}$ 和 Sakina ${ }^{[15,16]}$ 等人建立起来的研究陀螺自转轴进动的方法, 讨论在时空 (1) 中半径为 $r_{0}$ 的类时圆轨道上 陀螺的运动. 由于时空 (1) 具有球对称性, 不妨取轨道面位于 $\theta-\frac{\pi}{2}$. 由短程线方程得到陀螺 轨道坐标为

其中 $u$ 为固有时, 且

$$
\begin{aligned}
r=r_{0}, \theta & =\frac{\pi}{2}, \\
\varphi & =\sqrt{\frac{G M}{r_{0}^{3}}} \frac{u}{\sqrt{a-\frac{3 G M}{r_{0}}}}, t=\frac{u}{\sqrt{a-\frac{3 G M}{r_{0}}}},
\end{aligned}
$$

当把陀螺沿曲线 $C: x^{i}-x^{i}(u)$ 进行平移时, 设 $S^{i_{0}}$ 为 $u-u_{0}$ 时陀螺的自旋矢量, $S^{i}$ 为 $*=u$ 时刻自旋矢量, 得到 ${ }^{[1]]} \quad S^{j}=P_{i_{0}}^{i} S^{j_{0}}$,

式中 $P_{i}^{i}$ 称为平行传播子. 把 (4) 式代人短程线方程, 得到平行传播子满足方程:

$$
\frac{\mathrm{d} P_{i_{0}}^{i}}{\mathrm{~d} u}+\Gamma_{l k}^{\prime} \frac{\mathrm{d} x^{l}}{\mathrm{~d} u} P_{i_{0}}^{k}=0 \text {. }
$$

本文 1991 年 7 月 14 日收到. 1991 年 11 月 30 日收到修改稿.

* 圂家自然科学基金资助项目.

第 12 期

科学 通 报

1101 
把方柽 (1)、(2) 代人 (5) 式得到

$$
\begin{aligned}
& \frac{\mathrm{d} P_{i_{0}}^{1}}{\mathrm{~d} u}+\sqrt{\frac{G M}{r_{0}^{3}}} \frac{1}{\sqrt{a-\frac{3 G M}{r_{0}}}} \Gamma_{33}^{1} P_{i_{0}}^{3}+\frac{1}{\sqrt{a-\frac{3 G M}{r_{0}}}} \Gamma_{44}^{1} P_{i_{0}}^{1}=0, \\
& \frac{\mathrm{d} P_{i_{0}}^{2}}{\mathrm{~d} u}-0, \\
& \frac{\mathrm{d} P_{i_{0}}^{3}}{\mathrm{~d} u}+\sqrt{\frac{G M}{r_{0}^{3}}} \frac{1}{\sqrt{a-\frac{3 G M}{r_{0}}}} \Gamma_{31}^{3} P_{i_{0}}^{1}=0, \\
& \frac{\mathrm{d} P_{i_{0}}^{4}}{\mathrm{~d} u}+\frac{1}{\sqrt{a-\frac{3 G M}{r_{0}}}} \Gamma_{i_{1}}^{4} P_{i_{0}}^{1}-0,
\end{aligned}
$$

式中 $x^{1}-r, x^{2}-\theta, x^{3}=\varphi, x^{4}-t, j_{0}=1 、 2 、 3 、 4$. 方程 (6) 的解为

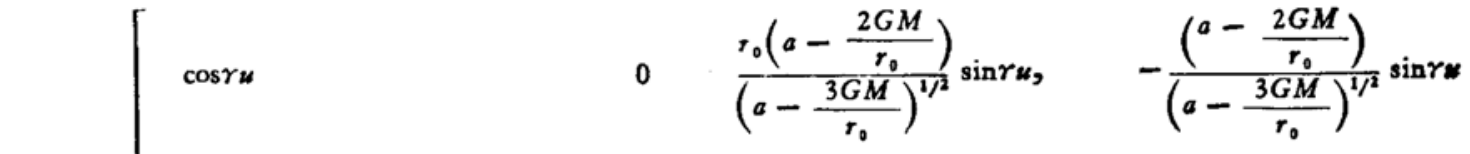

$$
\begin{aligned}
& 0
\end{aligned}
$$

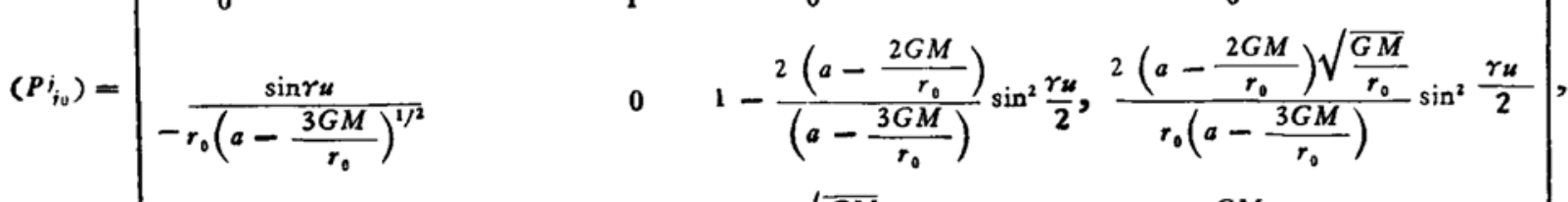

$$
\begin{aligned}
& -\frac{\sqrt{\frac{G M}{r_{0}}} \sin r u}{\left(a-\frac{2 G M}{r_{0}}\right)\left(a-\frac{3 G M}{r_{0}}\right)^{1 / 2}} \\
& 0 \quad-\frac{2 r_{0} \sqrt{\frac{\overline{G M}}{r_{0}}}}{a-\frac{3 G M}{r_{0}}} \sin ^{2} \frac{r_{u}}{2} \quad 1+\frac{2 \frac{G M}{r_{0}}}{a-\frac{3 G M}{r_{0}}} \sin ^{2} \frac{r_{u}}{2}
\end{aligned}
$$

式中 $r=\sqrt{\frac{G M}{r_{0}^{3}}}$. 为了使问题简化, 变换到陀螺共动坐标系, 为此, 引人正交标架 $\left\{\boldsymbol{e}_{\mathbf{a}}\right\}$ 及其 对偶基 $\left\{\omega^{\mathrm{a}}\right\}$ :

其中

$$
\begin{aligned}
& e_{i}=h_{i}^{k} e_{k}, \\
& \omega^{t}=t_{k}^{t} \omega^{k},
\end{aligned}
$$

$$
\left(h_{i}^{k}\right)=\left[\begin{array}{cccc}
\left\{e_{k}\right\}=\left\{\frac{\partial}{\partial x^{k}}\right\},\left\{\omega^{k}\right\}-\left\{\mathrm{d} x^{k}\right\}, \\
0 & \frac{1}{r_{0}} & 0 & 0 \\
0 & 0 & \frac{\left(a-\frac{2 G M}{r_{0}}\right)^{1 / 2}}{r_{0}\left(a-\frac{3 G M}{r_{0}}\right)^{1 / 2}} & \frac{\sqrt{\frac{G M}{r_{0}}}}{\left(a-\frac{2 G M}{r_{0}}\right)\left(a-\frac{3 G M}{r_{0}}\right.} \\
0 & 0 & \frac{\sqrt{\frac{G M}{r_{0}}}}{r_{0}\left(a-\frac{3 G M}{r_{0}}\right)^{1 / 2}} & \frac{1}{\left(a-\frac{3 G M}{r_{0}}\right)^{1 / 2}}
\end{array}\right.
$$




$$
\left(r_{k}^{i}\right)=\left[\begin{array}{cccc}
\frac{1}{\left(a-\frac{2 G M}{r_{0}}\right)^{1 / 2}} & 0 & 0 & 0 \\
0 & r_{0} & 0 & 0 \\
0 & 0 \frac{r_{0}\left(a-\frac{2 G M}{r_{0}}\right)^{1 / 2}}{\left(a-\frac{3 G M}{r_{0}}\right)^{1 / 2}} & -\frac{\sqrt{\frac{G M}{r_{0}}\left(a-\frac{2 G M}{r_{0}}\right)^{1 / 2}}}{\left(a-\frac{3 G M}{r_{0}}\right)^{1 / 2}} \\
0 & 0 & -\frac{r_{0} \frac{\sqrt{\frac{G M}{r_{0}}}}{\left(a-\frac{3 G M}{r_{0}}\right)^{1 / 2}}}{\left(a-\frac{2 G M}{r_{0}}\right)} & \frac{\left(a-\frac{3 G M}{r_{0}}\right)^{1 / 2}}{\left(a-\frac{1}{r_{0}}\right.}
\end{array}\right] .
$$

正交标架中平行传播子 $\boldsymbol{P}_{i_{0}}^{i}$ 与原坐标系中的 $\boldsymbol{P}_{i_{0}}^{i}$ 之间的变换方程为

$$
P_{i_{0}}^{i}=t_{k}^{i} h_{i_{0}}^{i_{0}} P_{i_{00}}^{k}
$$

把方程 (7)、(9) 和 (10) 代人上式得到

$$
\left(\hat{P}_{i_{0}}^{i}\right)=\left[\begin{array}{cccc}
\cos \gamma u & 0 & \sin \gamma u & 0 \\
0 & 1 & 0 & 0 \\
-\sin \gamma u & 0 & \cos \gamma u & 0 \\
0 & 0 & 0 & 1
\end{array}\right] .
$$

由于自旋矢量 $S^{i}$ 与四速度垂直, 即 $S_{i} \frac{\mathrm{d} x^{i}}{\mathrm{~d} \boldsymbol{u}}=0$, 把 $\mathrm{l}(2)$ 式代人得到

$$
S^{4}=\frac{r_{0} \sqrt{\frac{G M}{r_{0}}}}{a-\frac{2 G M}{r_{0}}} S^{3} .
$$

正交标架中自旋矢 $\hat{S}^{i}$ 与原坐标中 $S^{i}$ 满足变换式 $S^{i}=t_{k}^{i} S^{k}$, 于是

$$
\hat{S}\left(^{i}\right)=\left[\begin{array}{c}
\frac{1}{\left(a-\frac{2 G M}{r_{0}}\right)^{1 / 2}} S^{1} \\
j\left(a-\frac{3 G M}{r_{0} S^{2}}\right)^{1 / 2} \\
\frac{r_{0}\left(a-\frac{2 G M}{r_{0}}\right)^{1 / 2}}{(a} \\
0
\end{array}\right] .
$$

令 $\hat{S}^{i_{0}}$ 是在 $P_{0}$ 点 $(u-0)$ 的自旋矢, $S^{i}$ 是在 $P$ 点自旋矢, 设 $P$ 点是陀螺绕半径为 $r_{0}$ 的圆轨道转一周回到初始点处的位置, 则

$$
u=u_{p}=2 \pi r_{0}\left(\frac{r_{0}}{G M}\right)^{1 / 2}\left(a-\frac{3 G M}{r_{0}}\right)^{1 / 2} \text {. }
$$

由方程 (12) 知陀螺是绕 $\boldsymbol{e}_{\mathrm{i}}^{\hat{1}}$ 轴转动的, 所以不妨取 $\hat{S}^{2_{0}}=0$. 由矢量的夹角公式和 (15) 式得 到陀螺线圆轨道一周后, 其轴的进动角为 


$$
\delta=2 \pi\left[1-\left(a-\frac{3 G M}{r_{0}}\right)^{1 / 2}\right]
$$

相应的进动率为

$$
\begin{aligned}
\|\boldsymbol{Q}\| & =\frac{\delta}{u_{p}} \\
& =\frac{1}{r_{0}}\left(\frac{G M}{r_{0}}\right)^{1 / 2}\left[\left(a-\frac{3 G M}{r_{0}}\right)^{-\frac{1}{2}}-1\right] .
\end{aligned}
$$

当 $M / r_{0} \rightarrow 0$ 时, 进动角为

$$
\delta=2 \pi\left[1-\left(1-8 \pi G \eta^{2}\right)^{1 / 2}\right] .
$$

这表明: 当 $\eta \neq 0$ 时, 即使在很大的半径为 $r_{0}$ 的轨道上运动的陀螺, 绕轨道转一周回到出 发点,其进动角不为 0 . 当考虑最低级近似, (17) 式可写为

$$
\|\boldsymbol{\Omega}\|-\frac{1}{r_{0}}\left(\frac{G M}{r_{0}}\right)^{1 / 2}\left(\frac{3}{2} \frac{M}{r_{0}}+4 \pi G \eta^{2}\right) \text {. }
$$

上式比 Schiff 公式 ${ }^{[11]}$ 多出了右边第二项. 这表明当荷整体单极子后, 陀螺轴的进动率增大, 增大值与质量 $M$ 和对称破缺标度 $\eta$ 有关. 当令 $\eta=0$, 即不存在整体单极子, 方程 (16)一-(19) 退化为 Schwarzschild 时空中的结果. 这种差别出现的原因是: 当黑洞吞食了整体单极子, 其外部时空发生了根本的变化, 容易看出度规 (1) 在 $r_{0} \rightarrow \infty$ 时仍是非平直的.

应该指出的是：具有整体单极子黑洞的 Hawking 蒸发效应及其与通常 Schwarzschild 黑洞的 Hawking 蒸发效应的差异亦是一些很值得研究的问题 ${ }^{183}$.

\section{参考文献}

[1] Vilenkin, A., Phys. Rep., 121(1985), 263.

[ 2 ] Press, W. H. \& Spergel, D. N., Phys. Today, 42(1989), 29.

[ 3 ] Barriola, M. \& Vilenkin, A., Phys. Rev. Lett, 63(1989), 341.

[4] Yu Hongwei (余洪伟), Gen. Rel. Grav., 23(1991), 297.

[5] Jing Jiliang (制继良), J. Math. Phys., 32(1991), ! 1334.

[6] 余洪伟、王永久,科学通报,36(1991), 10: 734.

[7] 余洪伟、制继良、王永久，科学通报,36(1991),22: 1693 .

[8] Hiscock, W. A., Phys. Lett., B234(1990), 469.

[. 9] Mazzitelli, F. D., Phys. Rev., D43(1991), 468.

[10] Harari, D. \& Loustó, C. D., Phys. Rev., D42(1990), 2626.

[11] Schiff, L. I., Phys. Rev. Lett., 4(1960), 215.

[12] Everitt, C. W. F. et al., Experimental gravitation, Proceedings of the Intetrnational School of Physics Enrico Fermi, (Ed. Bereotti, B.), Varenna, Italy, 56(1974), 331

[13] Gonzalez-Martin, G., Phys. Rev., D14(1976), 399.

[14] Gonzalez-Martin, G., Phys. Rev., D17(1978), 972.

[15] Sakina, K. \& Chiba, J., Phys. Rev., D19(1979), 2280.

[16] Sakina, K. \& Chiba, J., Lett. Al. Nuovo Cimento, 27(1980), 184.

[17] Misner, C. W., Thorne, K. S. \& Wheeler, J. A., Gravitation, W. H. Freeman, San Francisco, 1973.

[18] Lousto, C., Ins. J. Mod. Phys. (to be published). 